Revista Brasileira de Meteorologia, v.27, n.3, 337 - 346, 2012

\title{
DISTRIBUIÇÃO ESPACIAL DA PRECIPITAÇÃO SOBRE O RIO GRANDE DO NORTE: ESTIMATIVAS VIA SATÉLITES E MEDIDAS POR PLUVIÔMETROS
}

\author{
CLÁUDIO MOISÉS SANTOS E SILVA ${ }^{1}$, PAULO SÉRGIO LÚCIO² ${ }^{2}$ MARIA HELENA CONSTANTINO \\ SPYRIDES ${ }^{2}$
}

\author{
${ }^{1}$ Universidade Federal do Rio Grande do Norte (UFRN), Departamento de Física Teórica e Experimental, \\ Natal, RN, Brasil \\ ${ }^{2}$ UFRN, Departamento de Estatística, Natal, RN, Brasil \\ claudio@dfte.ufrn.br, pslucio@ccet.ufrn.br, spyrides@ccet.ufrn.br
}

Recebido Maio de 2011 - Aceito Fevereiro 2012

\begin{abstract}
RESUMO
O objetivo deste estudo foi avaliar as estimativas de precipitação mensal fornecidas por duas fontes (algoritmo 3B43_V6 e Universidade de Delaware) para o Estado do Rio Grande do Norte, no período de 1998 a 2008. Utilizaram-se técnicas estatísticas baseadas em coeficiente de correlação linear e em índices de desempenho extraídos de uma tabela de contingência $2 \times 2$. Na análise, consideraram-se diferentes regimes pluviométricos, que são função da localização geográfica e do tipo de cobertura do solo. Constatou-se que o algoritmo 3B43_V6 subestimou a precipitação para a região Litorânea e superestimou a precipitação observada em regiões mais áridas (Seridó e Oeste). A precipitação de Delaware apresentou médias similares às observações, mas verificaram-se algumas discrepâncias aparentemente associadas ao método de interpolação e ao espaçamento de grade. Os resultados sugerem que ambos os produtos são capazes de representar variabilidades médias da precipitação mensal no espaço e no tempo; porém, apresentaram deficiências em identificar os eventos mais intensos de precipitação.
\end{abstract}

Palavras chave: 3B43_V6, Delaware, tabela de contingência, TRMM, semiárido.

\begin{abstract}
RAINFALL SPATIAL DISTRIBUTION OVER THE RIO GRANDE DO NORTE BRAZILIAN STATE: SATELLITES ESTIMATES AND RAIN GAUGES MEASUREMENTS

The aim of this study was to evaluate the monthly rainfall estimates obtained by two data sources (3B43_V6 algorithm and University of Delaware) for the State of Rio Grande do Norte, during the 1998 to 2008 period. Statistical analysis based on the linear correlation coefficient and on the performance index from a $2 \times 2$ contingency table was done. Coastland and semiarid precipitation regimes which depend on geographical location and on soil type coverage were considered. It was noted that the 3B43_V6 underestimated the coastland rainfall and overestimated the rainfall over arid zones. The Delaware mean rainfall agreed well with observed rainfall, but shows discrepancies concerning to interpolation method and grid spacing. The results suggests that both 3B43_V6 and Delaware rainfall dataset are able to capture the rainfall mean variability both in time and space; however, they show deficiency in identifing extreme precipitation events.
\end{abstract}

Keywords: 3B43_V6, Delaware, contingency table, TRMM, semiarid.

\section{INTRODUÇÃO}

O Nordeste Brasileiro (NEB) é caracterizado basicamente por três climas: litorâneo úmido (do litoral da Bahia ao litoral do Rio Grande do Norte); clima tropical úmido (em partes da Bahia, Ceará, Maranhão e Piauí); clima tropical semiárido (que abrange o sertão nordestino) (Kayano e Andreoli, 2009).
Fatores fisiográficos tal como relevo e a proximidade com a floresta Amazônica e a variabilidade e interação de sistemas meteorológicos em diferentes escalas de tempo e espaço são responsáveis por modular o clima do NEB. Em termos de precipitação pluviométrica Molion e Bernardo (2002) destacam a importância da migração meridional da Zona de Convergência Intertropical (ZCIT) como a principal forçante 
de grande escala sobre o NEB. Nos meses em que a posição da ZCIT está mais ao sul (março-abril), a porção norte do NEB apresenta máximos de precipitação; enquanto a posição da ZCIT mais ao norte (agosto-setembro) implica em escassez de chuva no norte do NEB.

A precipitação na faixa sudeste do NEB é influenciada pela penetração de sistemas frontais, que podem se organizar em uma faixa preferencial de convergência com orientação sudeste-noroeste, a chamada Zona de Convergência do Atlântico Sul (ZCAS); e também é afetada por sistemas convectivos de meso ou micro escala formados pelo mecanismo de brisa marítima. Na parte leste do NEB, o máximo de precipitação é observado entre junho e julho, período em que a ZCIT está muito próxima ou na sua posição mais ao norte; portanto, outros mecanismos são responsáveis por esse máximo de chuvas, entre eles destaca-se: a formação de uma zona de convergência na costa leste do NEB (Molion e Bernardo, 2002); perturbações ondulatórias ao longo dos alísios provenientes do Atlântico Sul (Torres e Ferreira, 2011); sistemas convectivos de mesoescala organizados em linhas de instabilidade (Cohen et al., 2009).

Especificamente sobre o Estado do Rio Grande do Norte $(\mathrm{RN})$, verifica-se uma distribuição espaço-temporal de chuvas bastante peculiar. No litoral a precipitação máxima ocorre em junho. Na parte central, o Seridó, que é uma região típica de semiárido e sofre com secas prolongadas, o mês de maior precipitação é março. Na faixa oeste, que faz parte do semiárido, o máximo de precipitação também ocorre em março; porém, apresenta algumas características de clima tropical úmido e, portanto, registra chuvas mais regulares e abundantes que o Seridó segundo dados da Empresa de Pesquisa Agropecuária do RN (EMPARN, 2010). Um dos resultados discutidos no último relatório do Intergovernmental Panel on Climate Change (AR4 - IPCC, 2007) foi sobre a possível transformação do semiárido nordestino em semideserto nos próximos 60 anos. Se de fato isso ocorrer o RN será muito afetado, pois apresenta cerca de 90\% de seu território com características de clima semiárido.

Nesse sentido, torna-se relevante avaliar produtos que forneçam o campo de precipitação sobre o $\mathrm{RN}$, pois essas estimativas podem ser usadas em estudos diagnósticos e prognósticos, especialmente em modelagem hidrometeorológica, buscando-se uma alternativa para os casos em que o monitoramento hidrometeorológico convencional é deficiente. Assim, o objetivo desse estudo é avaliar dois produtos de precipitação: o algoritmo 3B43_V6 do projeto Tropical Rainfall Measuring Mission (TRMM) e a precipitação observada sobre áreas continentais preparado pela Universidade de Delaware. A opção por se trabalhar com estes dois conjuntos de dados foi porque os dados da universidade de Delaware, originalmente, cobrem o período de 1900 a 2008 em uma grade de $0,5^{\circ}$, enquanto os dados do 3B43_V6, apesar de mais recentes, são dispostos em uma grade cujo espaçamento é de $0,25^{\circ}$. Logo esses dados podem ser úteis para estudos de variabilidade do clima de várias frequências, bem como, para a validação de modelos de circulação geral da atmosfera.

\section{METODOLOGIA}

\subsection{Precipitação estimada pelo TRMM}

Os instrumentos a bordo do satélite TRMM usados para estimar precipitação são: i) imageador de microondas (TMI); ii) radar de precipitação (PR); iii) radiômetro na faixa do visível e no infravermelho (VIRS). Além disso, o TRMM possui um sensor de energia radiante (CERES) e um sensor de imageamento de descargas elétricas em nuvens (LIS). O produto 3B43 V6 do projeto TRMM, usado neste trabalho, é uma estimativa mensal de precipitação que combina dois produtos: i) a estimativa a cada 3 horas do algoritmo 3B42_V6; ii) a precipitação observada por pluviômetros do projeto Global Precipitation Climatology Centre (GPCC). A estimativa 3B42 V6 é feita em duas fases. Na primeira, o algoritmo combina a precipitação estimada pelo TMI e o perfil de precipitação do PR, isso gera um produto mensal com espaçamento horizontal de $0,25^{\circ} \times 0,25^{\circ}$, que é chamado de $3 \mathrm{~B} 31$. Em seguida, o $3 \mathrm{~B} 41$ é usado para corrigir as estimativas de precipitação feitas através do satélite GOES, que é dada a cada 3 horas. Portanto, o produto 3B42 V6 apresenta espaçamento horizontal de $0,25^{\circ} \times 0,25^{\circ} \mathrm{e}$ frequência de 3 horas.

A precipitação do GPCC é resultado de um esforço internacional para criar uma análise global de precipitação com frequência mensal sobre o globo terrestre (Adler et al., 2003), para isso utilizaram-se 9.343 estações distribuídas pelos continentes. A vantagem do GPCC é que se trata de medidas de precipitação in situ, que a princípio não deve estar sujeita aos erros de estimativas dos satélites, interpoladas para uma grade de $1,0^{\circ} \times 1,0^{\circ}$ de espaçamento horizontal.

O produto 3B43_V6 é, portanto, uma estimativa de precipitação mensal que combina dados de satélites e medidas in situ sobre áreas continentais. A cobertura dos dados é de $50^{\circ} \mathrm{S}$ a $50^{\circ} \mathrm{N}$ em latitude e global em longitude. Apresenta espaçamento horizontal de $0,25^{\circ} \times 0,25^{\circ}$, ou seja, aproximadamente $25 \mathrm{~km}$ nos trópicos. Tem a vantagem de cobrir tanto áreas continentais quanto oceânicas, o que não é possível com os dados do GPCC.

\subsection{Precipitação interpolada pela Universidade de Delaware}

Os dados de estações para totais mensais de precipitação (mm) foram compilados a partir de várias fontes: dados do Global Historical Climatology Network (GHCN2), do 
Serviço Atmosférico e Ambiental do Canadá; do Instituto hidrometeorológico de São Petersburgo, Rússia (cortesia de Nikolay Shiklomanov); dados do projeto Greenland Climate Network, GC-Net (Steffen et al., 1996); registros a partir de estações meteorológicas automáticas do Projeto Groenlândia (cortesia de Charles R. Stearns na Universidade de WisconsinMadison), do Centro Nacional de Investigação Atmosférica (NCAR), dados diários da Índia; do arquivo de dados de precipitação do continente Africano, Nicholson (2001); dados mensais de precipitação do continente sul-americano, Webber e Willmott (1998); e do Global Surface Summary of Day (GSOD). O método de obtenção é descrito a seguir, sendo esquematizado na Figura 1.

Compararam-se os valores obtidos pelo GSOD com os de GHCN2, no período de 1951 a 1971, observando-se que os dados do GSOD tende a superestimar, sistematicamente, os valores correspondentes do obtido pelo GHCN2. Procedeu-se a uma interpolação geográfica dos dados do GHCN2 para a estimação dos dados correspondentes do GSOD. Se a diferença absoluta entre os valores estimados pelo GSOD e GHCN2 excedesse $80 \mathrm{~mm} \mathrm{mês}^{-1}$, o valor GSOD era substituído com o valor estimado pelo GHCN2. Outras estações climatológicas, como as utilizadas por Legates e Willmott (1990), também foram consideradas para compor a climatologia.

Utilizaram-se os valores de precipitação mensal das estações do GHCN2, por apresentarem um controle de qualidade.
Quando estes dados não estavam disponíveis utilizaram-se registros de outras estações próximas para compor a série daquela estação. Fez-se a imputação de dados com os registros de estações com as mesmas coordenadas geográficas. Quando uma estação continha pelo menos $90 \%$ de dados diários, estes foram utilizados para gerar os valores mensais. Caso contrário, se a estação continha dois ou mais dados, calculou-se a mediana. Se a estação só continha uma única informação, esta foi utilizada como a precipitação mensal. Dessa forma, aproveitou-se o máximo de informações disponíveis para compor a série, oriundas de um número de estações que variou entre 4.100 e 23.300.

\subsection{Precipitação medida no $\mathrm{RN}$}

Utilizou-se um conjunto composto de 21 estações nesse estudo para fins de validação. As estações são gerenciadas pela EMPARN e foram agrupadas nas três regiões: Litoral, Seridó e Oeste. Escolheram-se essas estações por não apresentarem falhas durante o período de análises, que foi de janeiro de 1998 a dezembro de 2008. A localização das estações é mostrada na Figura 2.

\subsection{Métodos}

As estimativas do TRMM e os dados da Universidade de Delaware foram interpolados por meio da média ponderada

\section{MAPA DO PROCESSO DE MONTAGEM DO BANCO DE DADOS DA UNIVERSIDADE DE DELAWARE}

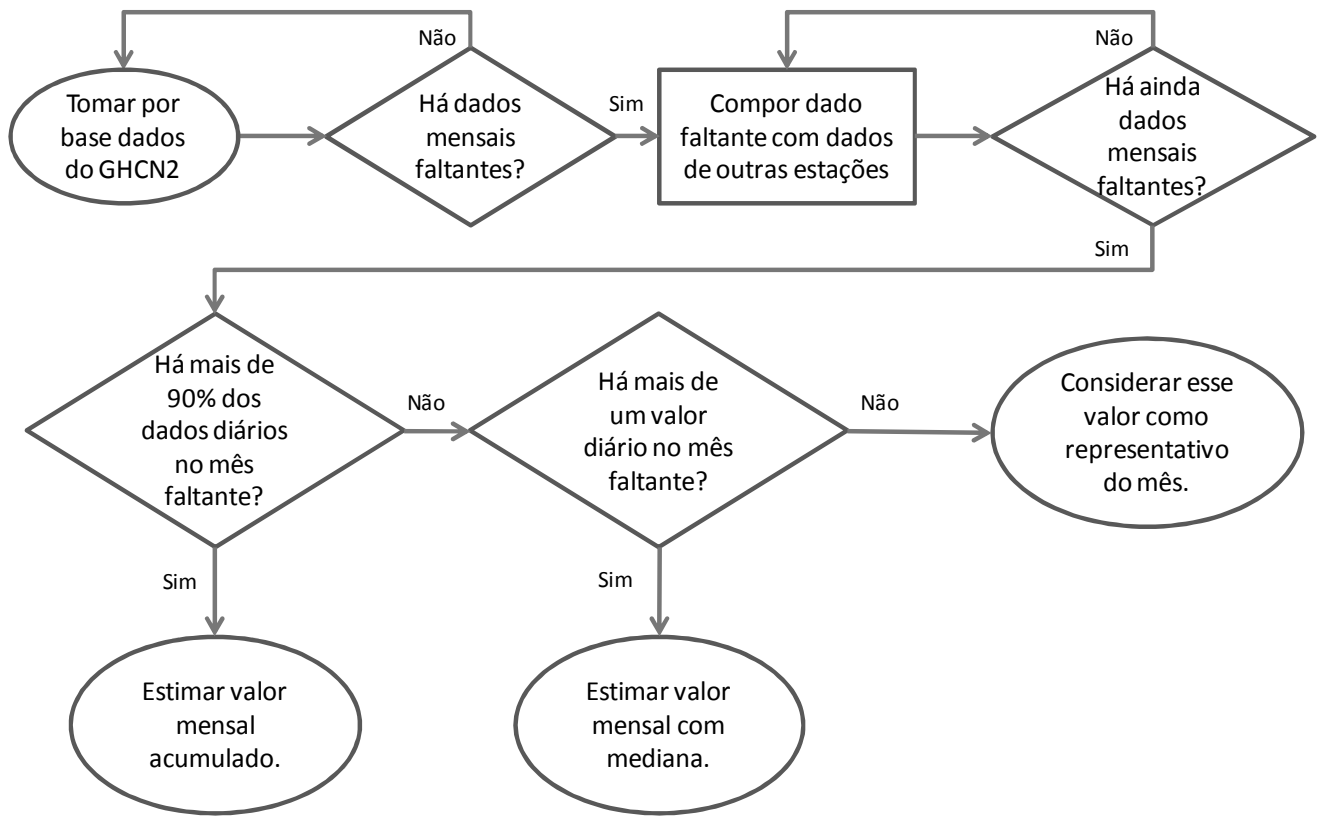

Figura 1 - Fluxograma que mostra o método de obtenção dos dados de precipitação da universidade de Delaware. 


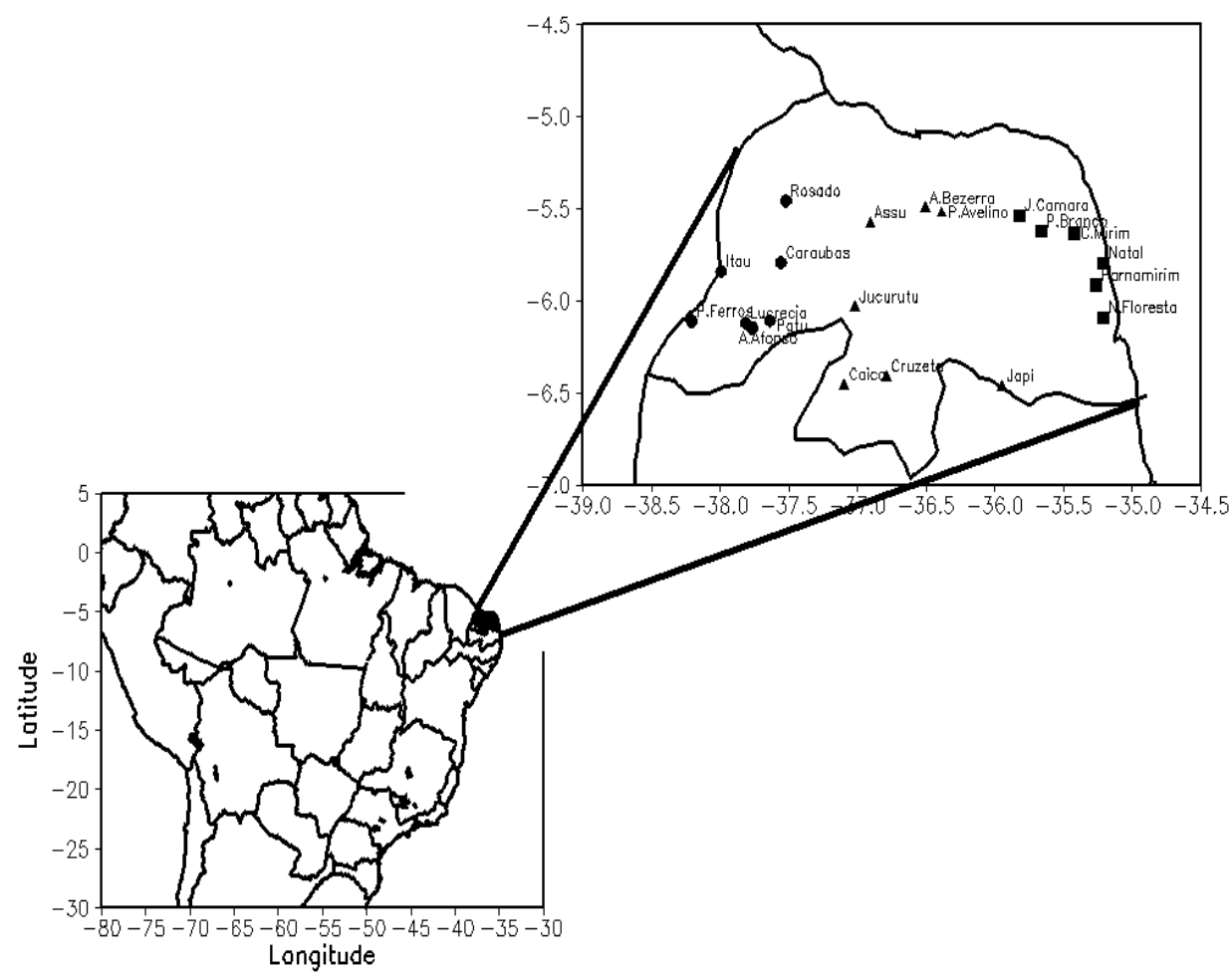

Figura 2 - Distribuição das estações de coleta de dados de precipitação no Estado do Rio Grande do Norte.

pelo inverso do quadrado da distância entre a estação de coleta de dados e os pontos de grade na qual a estação estava inserida, como a seguir:

$$
X_{p}=\frac{\sum_{i=1}^{4} \frac{1}{d_{i}^{2}} X_{i}}{\sum_{i=1}^{4} \frac{1}{d_{i}^{2}}}
$$

na qual $X_{p}$ é a precipitação interpolada para cada estação; $X_{i}$ é a precipitação nos pontos de grade; $d_{i}$ é a distância entre o ponto de grade e a estação; $i$ são os quatro vértices da grade na qual a estação está inserida. Calculou-se a média da precipitação medida e da precipitação interpolada para as estações de cada região considerada, analisando assim com base em três pares de séries. Aplicou-se o teste t de Student, seguindo Wilks (1995), para se determinar a significância estatística das correlações. Seja $t$ um valor tabelado em função da significância pretendida (no caso, 95\%), o teste das correlações é determinado pela expressão:

$$
t=\frac{r \sqrt{n-2}}{\sqrt{1-r^{2}}}
$$

Utilizou-se uma tabela de contingência $2 \times 2$ (Tabela 1) para avaliar as estimativas de precipitação sob diferentes limiares (Wilks, 1995), na qual “ $a$ " são os sucessos, "b" são os falsos alarmes, "c" são as perdas e " $d$ " são as negativas corretas (ou rejeições corretas). Os limiares estipulados para a determinação da tabela de contingência foram baseados nos quartis das observações. Assim, o primeiro limiar é o valor mínimo observado; o segundo limiar é o primeiro quartil; o terceiro limiar é o segundo quartil; o quarto limiar é o terceiro quartil; o quinto limiar é o quarto quartil. Após isso, definiu-se:

i) Probabilidade de detecção (POD): fração de acertos com relação ao total de casos observados

$$
P O D=\frac{a}{a+c}
$$

ii) Razão de falso alarme (RFA): fração de alertas falsos em relação ao total de ocorrências previstas

$R F A=\frac{b}{a+b}$

iii) Viés: razão entre o total de casos previstos e o total de casos observados (ou a razão entre as médias das previsões e a média das observações)

$$
\text { vies }=\frac{a+b}{a+c}
$$

Para a POD e a RFA o valor esperado é 1. Para o viés, valor maior que 1 indica superestimativa e menor que 1 , subestimativa. 


\section{RESULTADOS E DISCUSSÕES}

\subsection{Variabilidade mensal e inter anual}

O ciclo anual de precipitação estimada e observada nas três regiões (Litoral, Seridó e Oeste) é apresentado na Figura 3. Os maiores valores de precipitação foram observados no Litoral, que apresentou o máximo principal $\left(280 \mathrm{~mm} \mathrm{mês}^{-1}\right) \mathrm{em}$ junho, mínimo de $11 \mathrm{~mm}^{\text {mês }}{ }^{-1}$ em novembro e média de 114 $\mathrm{mm}$ mês $\mathrm{s}^{-1}$. Além disso, verificou-se que os meses de março a maio são caracterizados por máximos secundários, que apesar de não ultrapassarem $190 \mathrm{~mm}$ mês ${ }^{-1}$, representaram juntos cerca de $40 \%$ do total anual de precipitação. A precipitação média acumulada de março a julho corresponde a $75 \%$ do total anual da chuva observada. Os dois produtos de precipitação avaliados apresentaram ciclo anual muito semelhante entre si, embora o 3B43_V6 apresente valores ligeiramente superiores à precipitação de Delware, o que pode ser notado em janeiro, março, julho, outubro e novembro. Entretanto, no acumulado anual, a diferença foi de apenas $2 \%$ entre essas duas fontes de dados. Relativamente às observações, verificou-se que ambos subestimaram a precipitação e a diferença foi mais significativa no trimestre junho, julho e agosto. Além disso, o 3B43_V6 tendeu a superestimar a precipitação no trimestre março, abril e maio e durante o bimestre outubro, novembro.

A precipitação observada no Seridó apresentou máximo (140 $\left.\mathrm{mm} \mathrm{mês}^{-1}\right)$ em março e mínimo ( $\left.2 \mathrm{~mm} \mathrm{mês}^{-1}\right)$ em novembro e média de $51 \mathrm{~mm} \mathrm{mês}^{-1}$. A precipitação no primeiro semestre (janeiro a junho) representou $92 \%$ do total anual. A precipitação do 3B42_V6 foi sempre superior à precipitação observada e apresentou média de $85 \mathrm{~mm}$ mês ${ }^{-1}$, com $82 \%$ do total para o primeiro semestre, não obstante, o ciclo anual apresentou curvatura semelhante à precipitação observada através dos pluviômetros. A precipitação média de Delaware foi de $56 \mathrm{~mm}$ mês ${ }^{-1}$, com $86 \%$ registrada no primeiro semestre, um máximo secundário no mês de junho observado em consequência do método de interpolação e do espaçamento de grade de $0,5^{\circ}$ dessa base de dados. Na região Oeste a máxima $\left(188 \mathrm{~mm} \mathrm{mês}^{-1}\right)$ precipitação observada foi em março, a mínima $\left(2,5 \mathrm{~mm} \mathrm{mês}^{-1}\right)$ em novembro e a média $66 \mathrm{~mm} \mathrm{mês}^{-1}$. Registrou-se $92 \%$ da precipitação anual no primeiro semestre, semelhante ao Seridó.

Tabela 1 - Tabela de contingência 2x2.

\begin{tabular}{c|ccc}
\hline \multicolumn{3}{c}{ Observação } \\
\hline & & SIM & NÃO \\
\hline Estimativa & SIM & a & b \\
& NÃO & $c$ & d \\
\hline
\end{tabular}

Novamente o 3B43_V6 superestimou as observações, de forma que a média da precipitação desse algoritmo foi $88 \mathrm{~mm} \mathrm{mês}^{-1}$. A precipitação de Delaware também superestimou a precipitação observada, em função da precipitação elevada que essa base de dados registrou no mês de abril, porém a média foi mais próxima da observada, $70 \mathrm{~mm}$ mês $\mathrm{s}^{-1}$.

A característica comum entre precipitação do 3B43_V6 e a precipitação de Delaware foi a subestimativa no Litoral e superestimativa no Seridó e no Oeste. Porém, nessas duas últimas regiões, a precipitação do 3B43_V6 foi superior à precipitação de Delaware, ou seja, houve maior superestimativa por parte do produto do TRMM. Uma vez que a precipitação de Delaware são campos observados e interpolados para uma grade regular, as principais fontes de incertezas devem estar associadas ao próprio algoritmo de interpolação de dados e ao espaçamento de grade usado, que influencia diretamente o método de ponderação pelo inverso do quadrado (Equação 1). Por isso, a precipitação em junho no Seridó apresentou esse máximo visto a proximidade dessa região com o Litoral. De maneira semelhante, a precipitação de abril pode estar associada à interpolação da precipitação intensa em regiões mais a Norte, Sul ou Oeste. Sabe-se, por exemplo, que a precipitação máxima em Fortaleza-CE é em abril (Guedes et al., 2005) e isso pode ter influenciado a precipitação da região Oeste do RN.

Por outro lado, a precipitação do TRMM, embora utilize medidas diretas do GPCC, é baseada principalmente em dados de emissividade do sensor TMI e de refletividade do PR. Portanto, está sujeito também a erros de instrumentação. Em estudo sobre a África, Adeyewa e Nakamura (2003) verificaram que a precipitação do algoritmo 3B43_V6 apresenta ciclo sazonal muito semelhante, porém com ligeira superestimativa da precipitação observada. Além disso, esses autores verificam que os produtos baseados exclusivamente no PR tendem a superestimar a precipitação durante o ano todo. Não obstante, verificaram que a habilidade dos produtos do TRMM apresentou dependência regional, associado ao tipo de cobertura do solo.

A variabilidade mensal da precipitação é apresentada na Figura 4. Verifica-se que a precipitação intensa durante a estação chuvosa de 1999-2000 ocorreu em uma fase fria do El Nino Oscilação Sul (ENSO). Em 2004 ocorreu uma fase quente do ENSO, que em geral provoca escassez de chuva sobre o NEB, assim a precipitação intensa observada foi resultado de uma combinação de sistemas atmosféricos, tais como, a localização da ZCIT, incursões de frentes frias organizando sistemas convectivos de mesoescala, formação de vórtices ciclônicos de altos níveis, variabilidade intrasazonal, formação da ZCAS (Alves et al., 2006). Como resultado, a precipitação observada em junho desse ano sobre o Litoral (Figura 3a) foi superior a $350 \mathrm{~mm}$ mês ${ }^{-1}$, enquanto a precipitação do 3B43_V6 e de Delaware para esse mesmo episódio não 

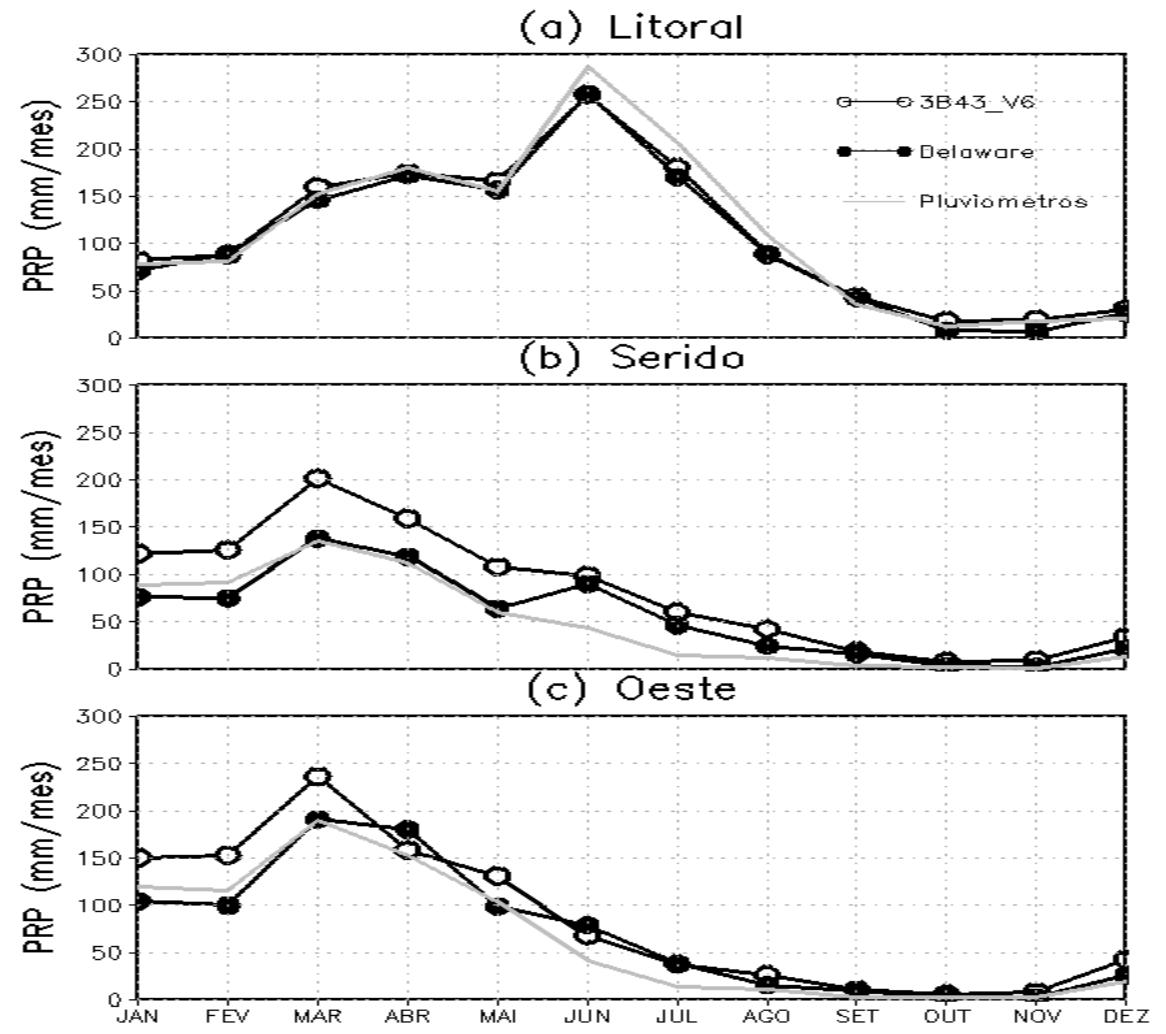

Figura 3 - Ciclo anual de precipitação dividido por regiões: (a) litoral, (b) Seridó e (c) Oeste.

ultrapassou $300 \mathrm{~mm}$ mês $^{-1}$. Já no evento de 1999-2000 a precipitação observada foi superestimada pelo 3B43_V6. Uma explicação para isso é concernente às escalas atmosféricas dos sistemas meteorológicos envolvidos, que em 2004 variaram desde a grande escala até a escala de cumulus; sendo assim, o espaçamento horizontal dos produtos analisados não foi suficiente para resolver processos das escalas menores e por isso subestimaram a precipitação. Outro detalhe foi a ocorrência de El niño moderado em 2002-2003 (http://enos.cptec.inpe.br/), que afetou a duração e a intensidade da estação chuvosa sobre a região e sendo assim, o máximo de precipitação observada foi em março e não ultrapassou $200 \mathrm{~mm}$ mês $^{-1}$.

No caso de 1999, houve predominância de sistemas de grande escala (Grimm e Tadeschi, 2009) e nesses casos uma das características dos produtos do TRMM é a superestimativa da precipitação (Huffman et al., 2007) devido a forte emissividade das nuvens que se organizam em grandes bandas estratiformes. Esse tipo de resposta dos produtos do TRMM também foi observado sobre a bacia amazônica, quando Santos e Silva et al. (2009) verificaram que a precipitação derivada do produto 3B42 V6 superestimou a precipitação medida por um radar no Estado de Rondônia durante eventos em que a ZCAS esteve configurada e atuando sobre essa região. Além disso, sobre outras bacias hidrográficas (Rio São Francisco, Bacia do Rio
Prata, Rios da Patagônia, Rio Araguaia, Rio Amazonas) a precipitação do TRMM tendeu a ser superior que a precipitação observada e simulada por modelos de circulação geral da atmosfera (Pinto et al., 2009).

\subsection{Análise estatística}

A primeira parte da análise estatística baseia-se em diagramas de dispersão e correlação linear, conforme mostrada na Figura 5. O 3B43_V6 apresentou melhores correlações em comparação aos dados de Delaware, porém apresenta diferenças entre as regiões estudadas. No Litoral a correlação foi 0,78 , aumentando para 0,89 e 0,93 no Seridó e no Oeste, respectivamente. Ao mesmo tempo o coeficiente angular da reta de regressão foi 0,78 (no Litoral) e 1,1 (no Seridó e no Oeste) indicando subestimativas na região mais úmida e superestimativa nas áreas mais secas. Também é possível verificar que a subestimativa no Litoral foi mais acentuada para eventos mais intensos de precipitação, a exemplo de dois casos em que as observações indicam chuva entre 550 e 580 $\mathrm{mm}$ mês $^{-1}$, ao passo que o 3B43_V6 acusou valores inferiores a $300 \mathrm{~mm}$ mês $^{-1}$. Em contrapartida, para valores abaixo de $200 \mathrm{~mm}$ mês $^{-1}$ a dispersão é menor. Com relação aos dados de Delaware, a menor correlação foi no Seridó $(0,61)$ que 

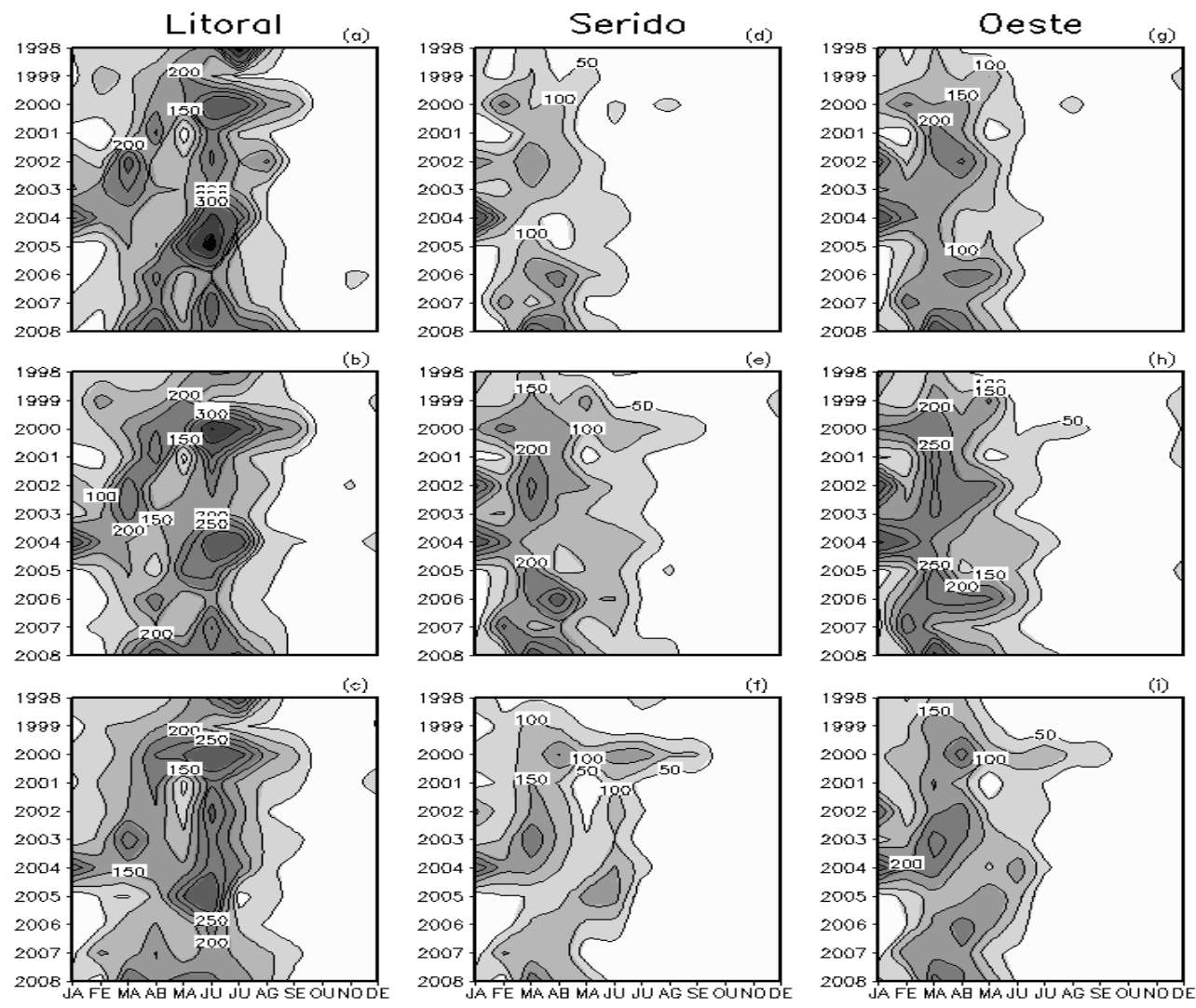

Figura 4 - Variabilidade mensal da precipitação. A primeira coluna é referente ao Litoral, a segunda ao Seridó e a terceira à região Oeste. A sequência a,d,g é para os dados dos pluviômetros; b,e,h referentes ao 3B43_V6; finalmente c,f,i são para os dados de Delaware. A unidade da precipitação é em mm com intervalo de $50 \mathrm{~mm}$, conforme indicado pelos contornos.

resulta do método de interpolação e espaçamento de grade conforme apresentado na Figura 3. Em todos os casos houve subestimativa da precipitação observada, porém, por se tratar de dados observados, a subestimativa dos valores extremos no Litoral foi menos pronunciada em comparação ao 3B43_V6.

A superestimativa do 3B43_V6 sobre regiões mais áridas, em comparação com regiões mais úmidas encontradas na presente pesquisa, é consistente com os resultados de Adeyewa e Nakamura (2003) que realizou estudos sobre áreas de diferentes tipos de cobertura vegetal na África. Além disso, as correlações medidas através do coeficiente $\mathrm{R}^{2}$ são coerentes com os obtidos sobre outras regiões, por exemplo: nos Estados Unidos (Novo México) (Chiu et al., 2006); sobre a Austrália, parte central dos Estados Unidos e na Inglaterra (Ebert et al., 2007); na Bacia Amazônica (Collischonn et al., 2008); na Grécia (Feidas, 2010); na Tailândia (Chokngamwong e Chiu, 2008); em Bangladesh (Islan e Uyeda, 2007). Sobre outras áreas da América do Sul, o desempenho dos produtos do TRMM (não necessariamente o 3B43_V6, mas a precipitação do PR) também apresentou resultados similares (Franchito et al., 2009), inclusive havendo alguns esforços para melhorar esse desempenho (Rozante et al., 2010).
A Tabela 2 apresenta os quartis das observações usados para o cálculo da POD, da RFA e do viés. Os valores correspondentes a Q0 são os mínimos registrados em cada região, logo nota-se que sobre o Litoral não houve mês sem chuva durante o período estudado em pelo menos um dos postos de coleta de dados. Ao contrário, no Seridó e no Oeste apenas acima do segundo quartil verificou-se precipitação acima de $18 \mathrm{~mm} \mathrm{mês}^{-1}$ e 22,8 $\mathrm{mm} \mathrm{mês}^{-1}$. Outro detalhe é que apesar da precipitação média acumulada no Seridó ser menor

Tabela 2 - Valor mínimo (Q0) e quartis dos dados de precipitação (mm mês-1) observados no Litoral, no Seridó e na parte Oeste do RN durante o período de estudo.

\begin{tabular}{cccc}
\hline & Litoral & Seridó & Oeste \\
\hline Q0 & 0,9 & 0,0 & 0,0 \\
Q1 & 24,1 & 2,8 & 2,4 \\
Q2 & 73,0 & 18,1 & 22,8 \\
Q3 & 157,5 & 72,8 & 109,2 \\
Q4 & 572,2 & 407,6 & 382,7 \\
\hline
\end{tabular}


(a)

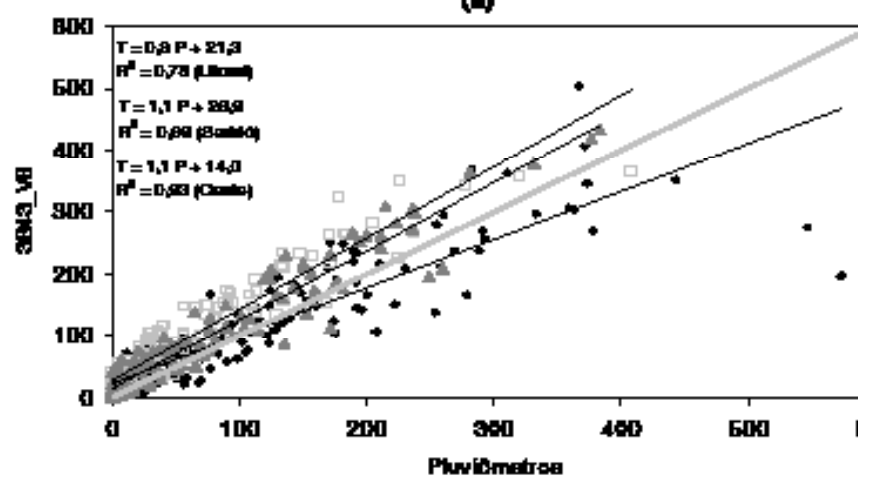

(b)

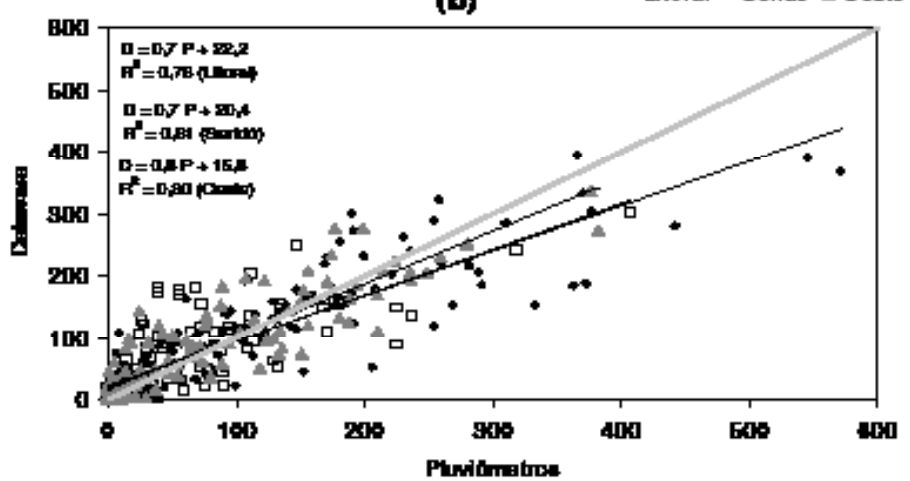

Figura 5 - Diagrama de dispersão entre as estimativas de precipitação do 3B43_V6 e a medida nos pluviômetros (a), dispersão entre os dados de precipitação da universidade de Delaware e a precipitação medida por pluviômetros (b). A precipitação é expressa em mm mês-1.

(a) Litoral

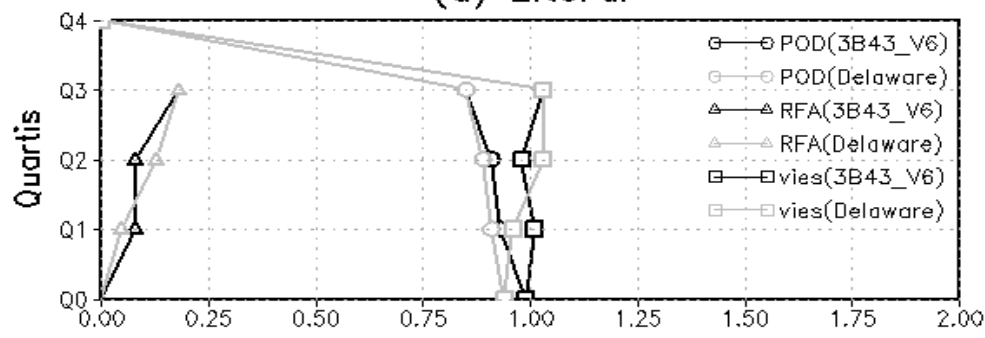

(b) Serida

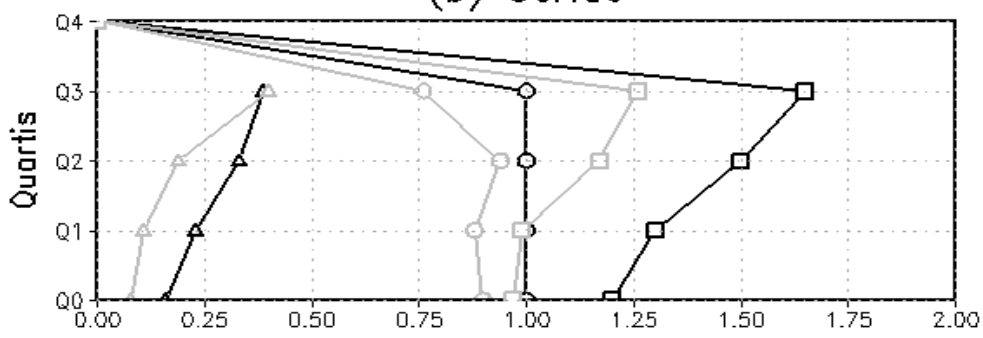

(c) Oeste

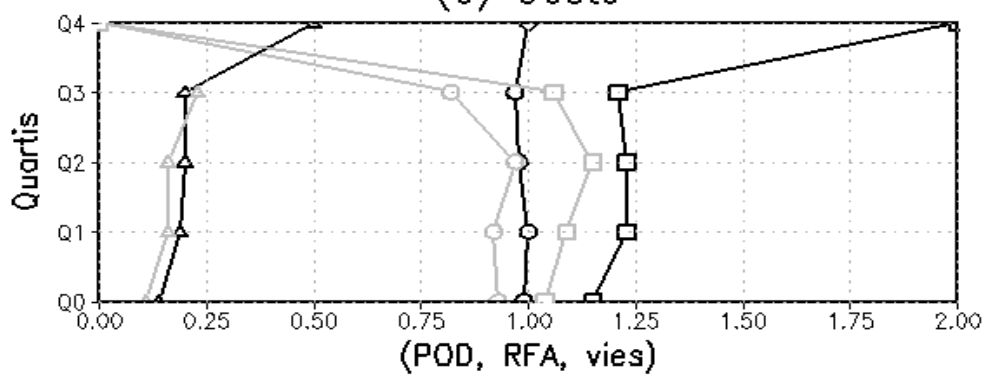

Figura 6 - POD, RFA e viés determinado através da tabela de contingência $2 \times 2$.

comparada às outras regiões, verifica-se que o quartil Q4 foi 407,6 $\mathrm{mm} \mathrm{mês}^{-1}$, enquanto o Q4 da região Oeste foi 382,7 mm mês ${ }^{-1}$, enfatizando a característica de má distribuição de chuvas sobre as regiões mais áridas do NEB, que hora sofre com secas prolongadas, hora são atingidas por eventos de chuvas excessivas (Kayano e Andreoli, 2009).
Os índices de desempenho extraídos da Tabela 1 são mostrados na Figura 6. No Litoral, a POD tendeu a diminuir com o aumento dos quartis, tornando-se nula para o quartil Q4, indicando que os métodos de obtenção dos dois conjuntos de dados não são hábeis em capturar os valores mais intensos de precipitação observada. No Seridó a POD diminuiu com o 
aumento dos limiares apenas para os dados de Delaware, embora haja um aumento no quartil Q2; no caso do 3B43_V6 a POD é constante e igual a 1 até o quartil Q3, diminuindo abruptamente para zero em Q4. Na região Oeste, a POD é próxima de $1 \mathrm{em}$ todos os quartis para os dados do 3B43 V6; para os dados de Delaware, novamente ocorreu um aumento em Q2, porém ocorreu diminuição até zero da POD para Q4. A RFA foi menor no Litoral, em que não houve valores maiores que 0,25 , e maior no Seridó para ambos os dados. Comparando as duas fontes de dados, observou-se que o 3B43_V6 apresentou os maiores valores de RFA, exceto para Q0 e Q1 no Litoral e para Q3 na região Oeste. Por sua vez, o viés dos dados de Delaware foi sempre menor que os dados do 3B43_V6 e apresentou superestimativa no Seridó e no Oeste e subestimativa somente para Q0 e Q1 no Litoral.

\section{COMENTÁRIOS FINAIS}

É crescente o número de produtos de precipitação estimados via satélites, bem como, existe um esforço científico em fornecer dados de precipitação interpolada para grades regulares com cobertura global. Exemplos são os dados do algoritmo 3B43_V6 e da precipitação da Universidade de Delaware. A precipitação pluviométrica é a principal variável do ciclo hidrometeorológico, e compreender suas variabilidades no espaço e no tempo são de suma importância, tanto para questões científicas, quanto para assuntos pertinentes à gestão de recursos hídricos. Dessa forma, é importante avaliar de forma objetiva as diferentes fontes de dados de precipitação, especialmente sobre o NEB, que é uma área de grande vulnerabilidade climática.

Assim, apresentou-se uma avaliação da precipitação do produto 3B43_V6 e da precipitação de Delaware. As duas fontes de dados foram eficazes em capturar, tanto a variabilidade espacial, quanto temporal, embora o 3B43_V6 tendesse a superestimar a precipitação mensal sobre as áreas mais secas e subestimar sobre as áreas mais chuvosas. Por sua vez, a precipitação de Delaware apresentou intensidade similar à precipitação média observada, pois se trata de um campo de precipitação interpolado para uma grade regular e, portanto, está sujeita a menos erros que as estimativas do 3B43_V6. As análises estatísticas empregadas apresentaram resultados consistentes com estudos realizados em outras partes do globo. Porém, um aspecto negativo dos dados foi quanto a pouca habilidade em representar meses de maior precipitação, contabilizados no quartil Q4 dos dados observados.

\section{REFERÊNCIAS BIBLIOGRÁFICAS}

ADEYEWA, Z.D. E NAKAMURA, K. Validation of TRMM radar rainfall data over major climatic regions in Africa,
Journal of Applied Meteorology, v. 42, n.2, p. 331-347, 2003.

ADLER, R. F. et al. The Version-2 Global Precipitation Climatology Project (GPCP) Monthly Precipitation analysis (1979-present), Journal of Hydrometeorology, v. 4, p. 1147 1167, 2003.

ALVES, J.M.B.; FERREIRA, F.F.; CAMPOS, J.N.B.; ASSIS, F.; SOUZA, E.S.; DURAN, B.; SERVAINI, J.; STUDART, T.M. Mecanismos atmosféricos associados à ocorrência de precipitação intensa sobre o Nordeste do Brasil durante janeiro de 2004, Revista Brasileira de Meteorologia, v. 21, p. 56-76, 2006.

CHIU, L.S.; LIU, Z.; VONGSAARD, J.; MORAIN, S.; BUDGE, A.; NEVILLE, P.; BALES, C. Comparision of TRMM and water district rain rates over New Mexico, Advances in Atmospheric Science, v. 23, p.1-13, 2006.

CHOKNGAMWONG, R.; CHIU, L.S. Thailand daily rainfall and comparison with TRMM products, Journal of Hydrometeorology, v. 9, p. 256-266, 2008.

COHEN, J.C.P.; CAVALCANTI, I.F.A.; BRAGA, R.H.M.; SANTOS NETO, L.A. Linhas de Instabilidade na Costa N-NE da America do Sul. In: CAVALCANTI, I.F.A.; FERREIRA, N.J.; SILVA, M.G.A.J.; SILVA DIAS, M.A.F. Tempo e clima do Brasil. 1.ed. São Paulo: Oficina de Textos, p. 75-93, 2009.

COLLISCHONN, B.; COLLISCHONN, W.; TUCCI, C.E.M. Daily hydrological modeling in the Amazon basin using TRMM rainfall estimates, Journal of Hydrology, v. 360, p. 207-216, 2008.

EBERT, E.E.; JANOWIAK, J.E.; KIDD, C. Comparison of near-real-time precipitation estimates from satellite observations and numerical models, Bulletin of the American Meteorological Society, v. 88, p. 47-64, 2007.

EMPRESA DE PESQUISA AGROPECUÁRIA DO RIO GRANDE DO NORTE (EMPARN): www.emparn.rn.gov. br, acesso em 26 de maio de 2010.

FEIDAS, H. Validation of satellite rainfall product over Greece, Theoretical and Applied Climatology, v. 99, p. 193-216, 2010.

FRANCHITO, S.H.; RAO, V.B.; VASQUES, A.C.; SANTO, C.M.E.; CONFORTE, J.C. Validation of TRMM PR Monthly rainfall over Brazil. Journal of Geophysical Research, v. 114, p. doi:10292007JD0, 2009.

GRIMM, A.M.; TADESCHI, R.G. ENSO and extreme rainfall events in South America, Journal of Climate, v. 22, p. 1589-1609, 2009.

GUEDES, R.L.; ANDREOLI, R.V.; KAYANO, M.T.; OYAMA, M.D.; ALVES, M.A.S. Série temporal de precipitação mensal de Fortaleza, Brasil: comparação entre observações e dados de reanálises do NCEP/NCAR. Revista Brasileira de Meteorologia, v. 20, p. 83-92, 2005. 
HUFFMAN, G.J.; ADLER, R.F.; BOLVIN, D.T.; GU, G.; NELKIN, E.J.; BOWMAN, K.; HONG, Y.; STOCKER, E.F.; WOLFF, D.B. The TRMM Multisatellite Precipitation Analysis (TMPA): quasi-global, multiyear, combinedsensor precipitation estimative at fine scales. Journal of Hydrometeorology, v. 8, p. 38-55, 2007.

INTERGOVERNMENTAL PANEL ON CLIMATE CHANGE (IPCC). The Scientific basis. Contribution of Working Group I to the Fourth Assessment Report of the Intergovernmental Panel on Climate Change. Cambridge, UK: University Press, 2007. 996f. Edited by Solomon et al.

ISLAN, M.N.; UYEDA, H. Use of TRMM in determining the climatic characteristics of rainfall over Bangladesh, Remote Sesing of Environment, v. 108, p.264-276, 2007.

KAYANO, M.T. E ANDREOLI, R.V. O clima da região Nordeste do Brasil. In: CAVALCANTI, I.F.A.; FERREIRA, N.J.; SILVA, M.G.A.J.; SILVA DIAS, M.A.F. Tempo e clima do Brasil. 1.ed. São Paulo: Oficina de Textos, p.213-233, 2009.

LEGATES, D.R.; WILLMOTT, C.J. Mean seasonal and spatial variability in gauge-corrected, global precipitation. International Journal of Climatology, v. 10, p. 111-127, 1990.

MOLION, L.C.B.; BERNARDO, S.O. Uma revisão da dinâmica das chuvas no Nordeste Brasileiro, Revista Brasileira de Meteorologia, v. 17, p.1-10, 2002.

NICHOLSON, S.E. A semi-quantitative, regional precipitation data set for studying African climates of the nineteenth century, Part I. Overview of the data set. Climate Change, v. 50, p. 317-353, 2001.
PINTO, L.I.C.; COSTA, M.H.; LIMA, F.Z.; DINIZ, L.M.F.; SEDIYAMA, G.C.; PRUSKI, F.F. Comparação de Produtos de Precipitação Para a América do Sul. Revista Brasileira de Meteorologia, v. 24, p. 461-477, 2009.

ROZANTE, J.R.; MOREIRA, D.S.; GONÇALVES, L.G.G.; VILA, D.A. Combining TRMM and Surface Observations of Precipitation: Technique and Validation over South America. Weather and Forecasting, v. 25, p. 885-894, 2010.

SANTOS E SILVA, C.M.; FREITAS, S.R.; GIELOW, R. BARROS, S.S. Evaluation of high-resolution precipitation estimate over the Amazon Basin. Atmospheric Science Letters, v.10, p. 273-278, 2009.

STEFFEN, K.; BOX, J.E.; ABDALATI, W. Greenland Climate Network: GC-Net, US Army Cold Regions Reattach and Engineering (CRREL), CRREL monograph, 1996.

TORRES, R.R.; FERREIRA, N.J. Case Studies of Easterly Wave Disturbances over Northeast Brazil Using the Eta Model. Weather and Forecasting, v. 26, p. 225-235, 2011. WEBber, S.R.; WILMOTT, C.J. South American precipitation: 1960-1990 gridded monthly time series (version 1.02), 1998. Disponível no site: http://climate. geog.udel.edu/climate, acesso em 04 de Julho de 2010.

WILKS, D.S. Statistical Methods in the Atmospheric Sciences: An Introduction. Academic Press: San Diego, CA, 1995. 